\title{
Using an Outliner With a Word Processor
}

\author{
Martha L. Hammel
}

Oakland University

Robert B. Kozma

\author{
NCRIPTAL \\ University of Michigan
}

\begin{abstract}
How do college students use computer-based outliners? What impact do these tools have on writing processes and products? This study examines the use of an outliner with a word processor by novice and advanced college writers. It also examines the impact of embedded prompts addressing topical and rhetorical issues. It shows how the outliner with embedded topical prompts serves both to increase the planning of novice writers and to connect this planning to their texts. These interventions are less effective for advanced writers. And the outliner without the prompts is also less effective for novice writers. Implications for classroom use of these tools are discussed.
\end{abstract}

Outlines have traditionally been considered a useful aid in the development of a written product. With the widespread use of computer-based writing tools, a growing number of word processors are designed with built-in outliners. But are these aids by themselves enough to help students who are learning to write? This article elaborates on a related study (Kozma, 1991b) to examine the impact of a computer outliner and embedded topical and rhetorical prompts on the writing processes and products of novice and advanced college writers.

Almost every composition text and handbook contains a section describing outlines (e.g., Fowler, 1980). The emphasis is frequently on the outline as an intermediate product that writers generate on the way to their final product - the compo-

The project presented, or reported herein, was performed pursuant to a Grant Number OERI-86-0010 from the Office of Educational Research and Improvement/Department of Education (OERI/ED). However, the opinions expressed herein do not necessarily reflect the position or policy of the OERI/ED and official endorsement by OERI/ED should not be inferred.

Requests for reprints should be addressed to Martha L. Hammel, Department of English, Oakland University, Rochester, MI 48309. 
sition. This product emphasis is expressed as a concern with the features and characteristics of a good outline. Traditionalists, for example, might emphasize a standard format (e.g., an introduction, body, and conclusion) or insist that an outline contain balanced entries (i.e., when an A appears, so must a B).

Alternatively, outlining may be viewed as a component of the more general process of writing. As such, it is not the form that an outline takes that is so important, but the underlying conceptual planning that goes into its construction. Outlining is one of various prewriting strategies that enable writers to express, examine, and modify their preliminary thinking. These strategies promote the generation of ideas and the arrangement of those ideas according to a specific purpose. Of the various prewriting strategies, outlining perhaps best promotes consideration of hierarchical relationships and the representation of these in linear form.

However, outlining may present some problems of its own for inexperienced writers. While outlining is frequently described as prewriting activity, experienced writers plan continuously throughout the writing process. These writers move recursively between planning, translating, and revising (Flower \& Hayes, 1981). Indeed, this transactional movement between text and plans is a dialectical process, called "knowledge transformation" by Bereiter and Scardamalia (1987), which results in both better plans and better text. Consequently, the process of outlining can best support writing if changes may be made to an initial outline at any time, even after the paper itself has been developed. But the outline may be viewed as "final" by less experienced writers, and it may foreclose the continuous revision of plans and overly constrain the emerging text.

Although advantages are claimed for outlines by composition texts, there is relatively little research on how writers actually use outlines and on the effectiveness of outlines on the quality of final compositions. Emig (1971) found that able high school writers voluntarily did little or no formal written planning, such as creating an outline. In an earlier pilot study in 1964 (reported in Emig, 1971), she examined two assumptions about outlining: (a) High school student writers do organize by outlining, and (b) student writers should organize by formal outlining in order to have a successful paper. She found that the first assumption was false, since only $9 \%$ of those in her study wrote outlines. Additionally, she found no correlation between the presence or absence of any outline and the grade the paper received for organization. On the other hand, Gee's (1984) study of 12th graders found a positive relationship between the use of outlines and rough drafts and the quality of written products.

More recently, an experimental study by Kellogg (1987) found that outlining by college students increased the amount of time they spend in prewriting and improved the quality of compositions. The students in this study who were required to outline produced longer writings, spent more time writing, and deleted more words while revising, compared to students who were not required to outline. They also wrote final compositions judged to be more effective and better developed than the compositions of students who did not outline. Outlining permitted a faster composition rate, though the overall efficiency of writing was not enhanced when the prewriting time needed for outlining was taken into account.

While few writers appear to use outlines (or they use them infrequently), initial research suggests that their use does influence planning and the quality of the composition. Our goal in this article is to extend this research to look at effects of computer-bascd outlining. Specifically, we are interested in how students use an outliner during the writing process and how computer-generated outlines are reflected in the 
final composition. We also examine how the use of topical and rhetorical prompts embedded in the outliner might affect the process and products of college writers.

\section{POTENTIAL BENEFITS OF COMPUTER-BASED OUTLINERS}

A computer-based outliner combines the organizational power of the traditional outline with the flexibility and fluidity of electronic text. The functions of these tools correspond to some of the cognitive needs of writers, particularly novice writers who can benefit from thinking more about the higher level structure of the text and about their writing plans (Kozma, 1991a). By supplying some of the surface structures of the outline and supporting some of the cognitive requirements of writing, a computer-based outliner may shift the writer's focus from the outline as a product to outlining as a planning process.

For example, computer outliners provide the "form" or template of the outline, and prompt the student to supply the ideas. Supplying the form eliminates the cognitive demands associated with recalling rules of proper outline form (e.g., "Do I use a Roman numeral or an Arabic numeral for this heading?"), demands that can conflict with cognitive resources needed for the planning process. The act of filling out this form may help writers sort out their initial, random ideas and build relationships among them. Deciding where to put each idea as it comes may require the writer to examine how the idea relates to all the other ideas that have come before. Used in this way, outliners may assist not only in the generation of ideas but also in the analysis of those ideas (Wresch, Pattow, \& Gifford, 1988).

Other features of the outliner may reduce the perception of the outline as a fixed structure and support the view of outlining as a dynamic, interactive process. For example, the ability to collapse or expand an outline allows the writer to temporarily put aside distracting evidence or supporting points while deciding the organization of the primary ideas. The program also makes it easy to move an entire section of the outline or move an entry into a subordinate or superordinate position relative to another idea. Thus, content, structure, and subordination can be easily revised at any point, promoting a more iterative approach to the outlining process and a more flexible use of the outline.

Such features in an outliner may increase the amount of time spent in planning before writing begins or the amount of time spent revising plans as writing progresses. While Haas (1989) found that the amount of planning decreased when word processors alone were used, perhaps the addition of an outliner to a word processor will counteract this effect.

On the other hand, the mere provision of empty "slots" in a template to be filled by the writer may be insufficient support for novices, those still struggling with the writing process. While outliners provide tools to make it easier to manipulate ideas, they provide no overt guidance in what those ideas might be. Novice writers may need additional support in the consideration of topical and rhetorical issues, support that might come from the use of prompts embedded in the software (Kozma, 1991a). The provision of this kind of ideational guidance, or scaffolding (Brown \& Palinscar, 1989), may extend the user's current capabilities and provide a model for skills that can be internalized with use.

The present study examines the use of a computer-based outliner, with and without embedded topical and rhetorical prompts, and its impact on the processes and 
products of novice and advanced college writers. It examines the reciprocal relationship between the skills and abilities of the user and the capabilities and design of the software (Kozma, 1991c; Salomon, Perkins, \& Globerson, 1991). Specifically, the following questions are addressed:

1. Do novice writers use the features of an outliner and embedded prompts differently from experienced writers, and are their processes and products differentially influenced?

2. What features and functions of an outliner are used by writers?

3. How does the use of an outliner affect planning?

4. How do topical and rhetorical prompts embedded in the outliner influence the writing process?

5. How does the use of an outliner and the prompts affect the structure and content of the final product; that is, what relationships exist between the outline and the essay?

\section{RELATED STUDY}

This article describes the ways college writers used a computer-based outliner, both with and without topical and rhetorical prompts. It is part of a larger, comparative study (Kozma, 1991b) involving 41 students. In the larger study, a third of the students used an outliner with a word processor, a third used a graphic idea organizer with a word processor, and another third used only a word processor. Half of the students in each group were given rhetorical and topical prompts and half were not. The study examined the impact of these tools both on the writing process and on the quality of the compositions of novice and advanced writers. Measures of the writing process were derived from think-aloud protocols that were transcribed and coded by trained staff for instances of specific cognitive behaviors, such as planning, evaluating, writing, and so on. However, the written prompt responses were not coded as planning so as not to inflate the frequency of this code. The quality of the compositions was assessed with a primary-traits scale used by highly trained and experienced raters on the staff of a writing assessment center.

In brief, it was found that both the outliner and the idea organizer resulted in more conceptual planning, when compared with the use of the word processor alone. This kind of planning involves the consideration of structure, rhetorical goals, and the writing process itself - the sort of planning associated with more experienced writers (Haas, 1989). The students who used prompts also did more conceptual planning than those who did not. This increased planning, however, resulted in better compositions only under certain conditions: Novice writers using the outliner with prompts wrote the best essays among all groups of novice writers. On the other hand, advanced writers using the outliner wrote the worst papers among advanced writers, particularly when accompanied by embedded prompts. Why were there these differences in the quality of compositions when all of these writers increased their planning with the use of these aids?

This article takes a closer look at the use of the computer-based outliner and embedded prompts by these students. It looks at how novice and advanced writers used the outliner differently. It examines how this use was influenced by embedded topical and rhetorical prompts. And it examines the relationship between user, tool, and task to identify the contributions of each to the resulting processes and products. 


\section{THE OUTLINER}

The software used in this study was Acta, version 2.0, and MacWrite, version 4.5. Acta is an outliner/desk accessory available for the Macintosh. It has many features now built into some word processors, such as Microsoft Word 4.0. Its primary features include a virtually unlimited number of topics, ample available space within each topic, cut-and-paste movements of text (both within Acta and into MacWrite), and easy collapse/expand, and easy movement of topics to other positions in the outline or levels of subordination. The entries and levels of subordination are signified by markers (i.e., arrows) and indentation rather than by standard outline notation (i.e., Roman numerals, capital letters, etc.). MacWrite, version 4.5, is a basic word processor with standard features that allow easy text entry and manipulation. (It has since been replaced by Claris MacWrite II, which has additional features, such as the availability of multiple windows, a built-in spell checker, and on-line help, although it does not have a built-in outliner.)

A particularly attractive advantage that $\Lambda$ cta offers is the availability of multiple windows. As many as four Acta windows can be open on the screen at the same time in addition to a MacWrite window. Movement between windows as well as into and out of MacWrite is easily accomplished by a click of the mouse anywhere on the desired window, thus making these two packages virtually integrated into one. This click activates the window, bringing it forward on the screen. The simplest arrangement of the windows is to have an open MacWrite window "on the bottom" with the Acta windows arranged on top (as illustrated in Figure 1). Further, shrinking the MacWrite window by one-half in. affords the students space to arrange the windows so that some edge of each window is visible at all times. This makes it easy to find and activate any window. The use of multiple windows

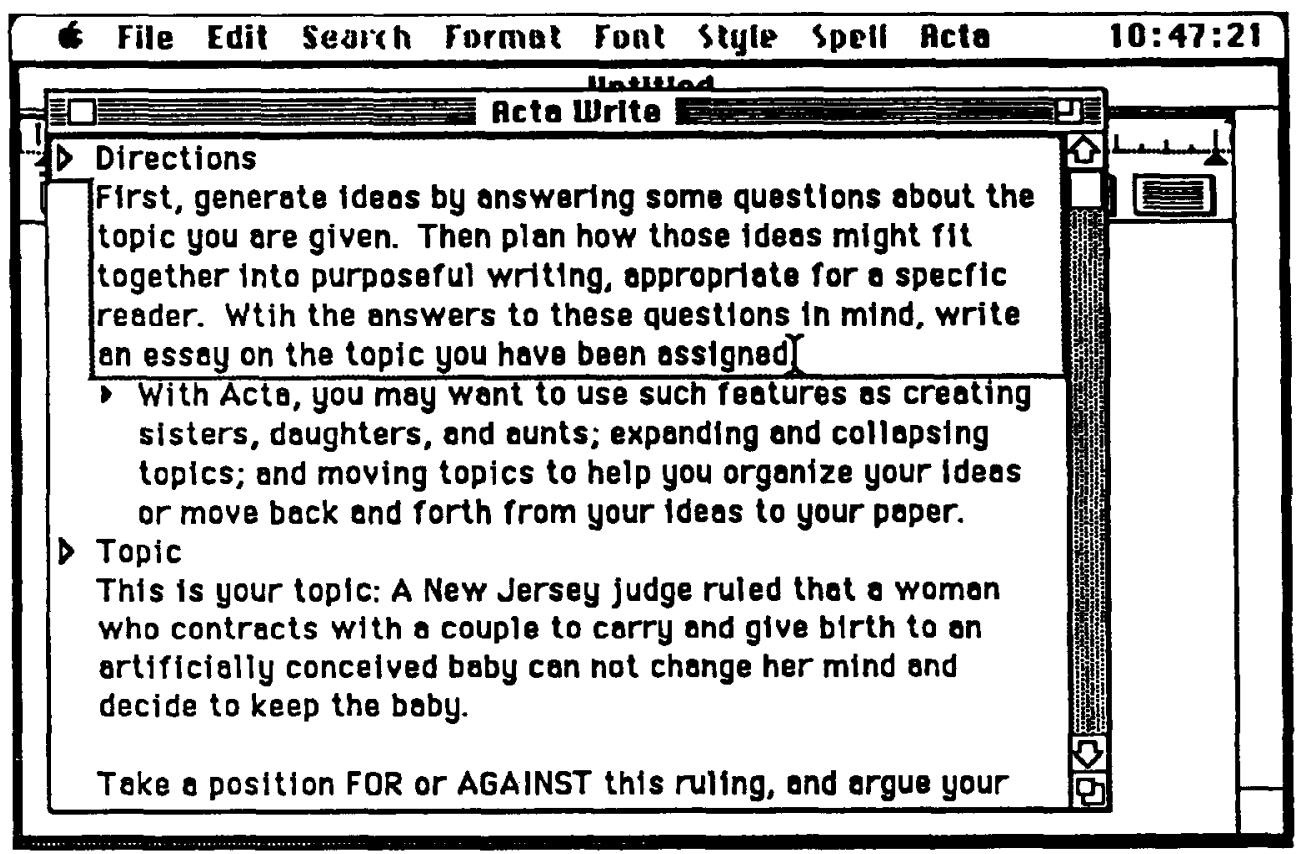

Figure 1. Screen from Acta with embedded prompts. 
make it possible to embed the topical and rhetorical prompts in one Acta window, have the students construct the outline of the paper in another Acta window, and write their composition in MacWrite.

\section{THE PROMPTS}

The prompts were designed to encourage writers to recall, analyze, and integrate topical information; to analyze the predispositions and expectations of the intended readers; and to analyze the relationship between topic and audience and express this as a goal statement for the composition. These prompts are listed in detail in Appendix A. For those that received them, the prompts were embedded as entries in the outliner such that each prompt heading (e.g., Your topic, Your reader, etc.) was a top-level entry and questions under each heading were positioned as subordinate entries. Thus, the user could collapse the prompts under the headings, although the prompts were presented to the subjects in the expanded form. This, coupled with the integrated arrangement of Acta and MacWrite, allowed the subjects the flexibility to enter their responses to the prompts in the outliner right next to question, they could create another outline for their responses, or they could enter their ideas directly into MacWrite.

\section{PROCEDURES}

\section{Subjects}

The subjects in the current study are the five novices and five advanced writers who used the outliner in the larger, related study (Kozma, 1991b). Volunteers in the larger study were recruited from among experienced Macintosh computer users. Student experience with computers ranged from less than a year to 3 or more years, with the modal response being 3 or more years of experience with computers. Students used the computers primarily for word processing, with the modal frequency of use being once a week.

The novice writers were students enrolled in an introductory English composition course at a midwestern comprehensive university. The advanced writers were students at a midwestern research university who had taken at least two writing courses, one being a course in argumentative writing. Consequently, the novice writers tended to be freshmen or sophomores taking their first writing course, while the advanced writers tended to be juniors and seniors who had taken an average of 4.1 literature and composition courses. Unsurprisingly, the quality of baseline compositions, as rated by trained evaluators, was significantly better for the advanced writers than for the novices. Advanced writers also typed better. In other respects, these students were similar, including self-reported grade point average and computer experience.

\section{Methodology}

The 10 students examined in this report were those randomly assigned to the outliner in the larger study (Kozma, 1991b) and selected to have their think-aloud protocols transcribed and analyzed by trained coders. Five of these subjects ( 3 of the novices and 2 of the advanced writers) were assigned to use the prompts embedded 
in the outliner; the others ( 2 novices and 3 advanced writers) received the outliner and word processor without prompts. Subjects who were not given embedded prompts did receive the outliner with an entry that stated the assigned topic for their paper (see Figure 1). They had access to all of the features of the outliner, but there was no other information embedded in the outliner.

As mentioned, all students recruited for the study had at least some prior experience with the Macintosh computer. In addition, all received a 1 1/2-hr training session on how to use the word processor and the outliner. During the training session, each of the software's functions was demonstrated and students were instructed to practice their use. The functions of the outliner covered during the training are listed in Appendix B. During a subsequent session, students practiced their use of these functions while they wrote a composition similar to the one used as the final task. They were told to use each of the functions covered during the training session and their ability to do this was monitored. Thus, subjects had a total of approximately $31 / 2 \mathrm{hr}$ of instruction and monitored-practice using the software over two sessions.

Two hours were scheduled for cach writing assignment. Most students were finished after an hour and a half, and no students were interrupted before they were finished. Each of the assignments was an argumentative task. Students were given a controversial court decision and asked to take a position for or against the ruling and support their position. For example, the final assignment was:

A New Jersey judge ruled that a woman who contracts with a couple to carry and give birth to an artificially conceived baby can not change her mind and decide to keep the baby. Take a position for or against this ruling and argue your position convincingly.

\section{Analysis of the Data}

Think-aloud protocols provide important data for analyzing cognitive processes (Hayes \& Flower, 1980). Prior to the session, all of the students were trained to "think aloud" while they worked. These statements were audio-recorded and transcribed.

Students' think-aloud protocols, notes, prompt responses, and compositions were analyzed. These data were examined using the constant comparative method of qualitative analysis described by Glaser and Strauss (1967; Glaser, 1978). This iterative approach begins by analyzing cases to identify and progressively elaborate and refine common patterns of cognitive behavior within subgroups of subjects. Subsequent analysis identifies differences between groups and establishes, tests, and qualifies propositions and relationships among variables.

\section{RESULTS}

\section{Novice Writers With Prompts}

Novice writers using the outliner with prompts wrote the best compositions of all novices in the larger study. How did they use the prompts and outliner to help them write better compositions? An analysis of the think-aloud protocols indicates that the prompts played a crucial role for this group. The prompts aided the consideration of content issues, and either the outline they generated in response to the 
prompts or the embedded prompts themselves served as a structural device to organize this content.

At the beginning of the session, Robert (all names are fictitious) read through all of the prompts without responding. He went back and reread the topical prompts and made a brief outline of four entries and $\mathbf{3 1}$ words in a second Acta window. No details or subordination appears in the outline.

However, in Robert's case, it was the embedded prompts rather than the outline that provided structural guidance for his composition. Having completed his brief outline, he then moved into MacWrite, saying, "Okay, I'm drawing a blank right now. Okay, at this point just start writing and figure it out." The outline seemed to offer little assistance, and Robert did not return to it once he began writing his essay. Rather, he made regular use of the prompts, specifically the topic prompts. He did not respond to any of the reader or goal prompts. After writing a few sentences in his MacWrite file in response to a prompt, Robert returned to read the next prompt, responding to it again in his text file. He referred to the topic prompts four times in this way, and these helped guide the generation and sequence of topical information that appeared in the final text.

For example, after beginning the composition by restating the court decision, Robert goes on:

\footnotetext{
Okay, let's get back to Write [the name of the Acta file with the embedded prompts]. I've restated the topic [one of the prompts]. [Reading the next prompt:] Make a list of the reasons this might be a good decision. Let's see. [Writing in his text file:] This decision was a good because it leaves the child in the best home environment while future courts . . Let's see. [Rereading:] The decision was a good one because it leaves the child in the best home environment while future courts decide on the point of law on which the case will hinge.
}

There was a direct correspondence between the topical prompts and the structure of his final essay.

As did Robert, Stacy began the session by reading all of the prompts straight through. She then went back to answer each prompt in a second Acta window. The order of the outline was exactly the same as the order of the prompts. Unlike Robert, Stacy responded extensively to the reader and goal prompts. In fact, she was the only novice that made any use of these prompts.

The outline Stacy created was the longest of all the Acta users, 30 entries long, with four top-level entries and as many as four subordinate levels. However, despite her extended use of the outliner, the only functions she employed were those to generate entries. She did not use the functions for expanding or contracting the outline or moving entries.

Once she finished responding to the prompts, Stacy began her composition with the first paragraph. She reread the assignment and added a second paragraph. She moved back and forth between her composition and the prompts or her outline three more times, occasionally adding text to her essay. However, it was the prompts rather than her outline that had a greater effect on her final product. Most of the text was written after reading the embedded prompts - specifically, the topic and goal prompts. After generating most of the final text, Stacy used the prompts to review her composition. There was little correspondence between the essay and her outline. It was referred to only once while Stacy was writing, and the reference did not result in the generation of additional text.

Another novice, Jean, also began the session by reading through all of the prompts. She exhibited the most sophisticated use of notes and outlines as part of 
her planning process. She reread the first two topical prompts and wrote some notes on paper under the headings "Pro" and "Con." She then reread her paper responses and the topical prompts and created the following outline:

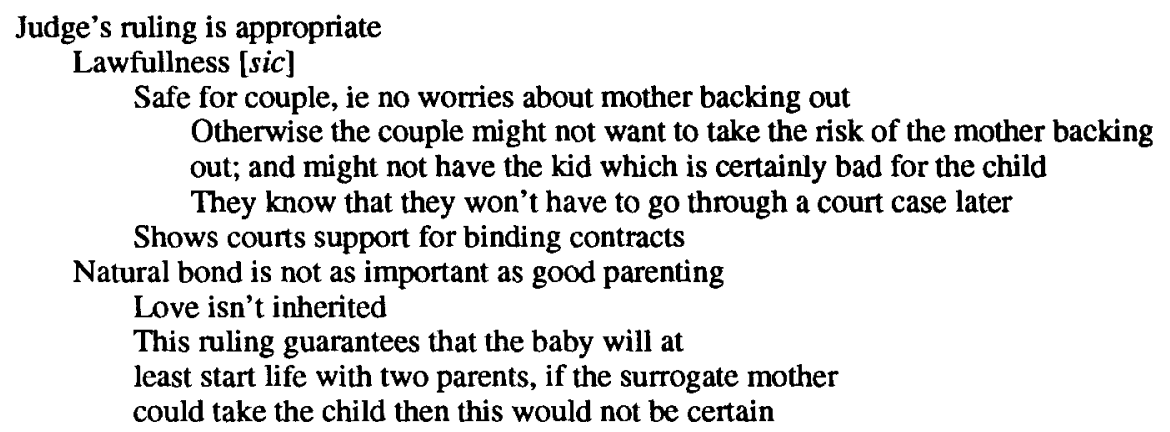

The arguments under the "Lawfullness" entry of her outline are those listed under the "Pro" section of her paper notes. The arguments under the "Natural bond" entry were converted into "Pro" rebuttals of arguments listed under the "Con" section of her paper notes.

As Jean started writing her composition, she arranged windows on the screen to "make it easy to flip back and forth" between the outline and her composition. She moved between her outline and the composition four times while writing, and there is a direct correspondence between the outline and the sequence of generated text. Indeed, twice she copied and pasted sentences from her outline directly into her final text.

For novice writers, the prompts served a critical function. But it was the topic prompts that helped them consider their position on the issue and generate information that appeared in the final text. The reader and goal prompts were used by only one novice. An outline was used to organize this content information. Two of the novices made brief outlines and one created an extended outline; but none of the novices used the advanced functions of the outliner. Subsequent to initial planning, either the outline generated in response to the prompts or the embedded prompts themselves served to guide the generation of the final text. Robert and Stacy relied primarily on the prompts to guide their composition, while Jean used the outline she created in response to the prompts and her paper notes. For these novice writers, the outliner with prompts seemed to foster the generation of more plans and serve as an effective bridge between planning and the final composition.

\section{Novice Writers Without Prompts}

But, how important were the prompts in this process? How do novices use an outliner by itself? Is it a useful aid for novice writers if it is unaccompanied by prompts? The two novices examined here used the outliner in different ways to help them with their planning. For these students, however, increased planning did not result in better compositions.

Mary used the outliner to write the major ideas for the essay as primary level statements. The outline was moderately developed, with 113 words, but it did not show much hierarchical structuring of ideas. She made four top-level entries; one of them had two supporting, subordinate entries. Mary did not use the expansion or contraction features of Acta, but did use it to change the order of some of the statements. 
There is a direct correspondence between Mary's outline and her composition; each one of the top-level entries in Mary's outline corresponded to a topic sentence of a paragraph in her text. However, what is of interest is the sequence of development of the outline and the composition. The outline entries were not made prior to starting the composition. Mary began by writing the sentence that appears first in the final composition. She then moved to Acta and created her first entry, returning to her composition to develop the idea. She returned to her outline two more times, making an entry that corresponded to ideas she had already composed in her text. For Mary, the outline seemed to be an afterthought, an exercise that needed to be done in order to complete her assignment. It did not seem to provide her with an opportunity to think about her topic in a preliminary way.

Mary's use of the outliner can be contrasted with that of Janice, another novice. Janice began by saying, "Well, this is a topic that I don't have a decision for, so I think I'll start my regular outline of the pros and cons." This suggests that writing an outline was a habit for this student. She made nine entries: three top-level entries and six subordinate entries. One top-level entry was a restatement of the issue, and the others were headings for the pro and con arguments. These were entered in sequence. As in the case of Mary, Janice did not use any special features of Acta. She did not change the order of any ideas once written in Acta nor did she cut and paste anything into MacWrite.

In the course of listing both sides, Janice seemed to sort out her thinking while using the outliner. At the end of the listing, she again argues the pros and the cons with herself and concludes:

Uh, let's see, so I think l'll start writing the paper, and I'm gonna take the position of being against the mother being able to change her mind ...

With this position in mind, Janice begins writing the composition. However, she does not start her writing with the first point in her outline in mind. Instead, she decides to write "a story of something I heard." At this point the composition evolves out of the text, and writing progresses in a write-reread-write fashion. About halfway through the essay, Janice returned briefly to Acta to check arguments on the con side of the outline, but did not change anything in Acta, nor does the point that she reviewed appear in the text. In general, the composition did not correspond to the outline; not only did points that appear in the outline not end up in the text, several points made in the text are not in the outline. For Janice, the outlining process proved to be a prewriting exercise important to clarifying her position on the issue. Beyond this, the outline had little connection to the final product, nor was it used effectively while the essay was composed.

Novice writers in the larger study who used the outliner did more planning than those who used the word processor alone, but their compositions were better only if accompanied by embedded prompts. While it is clear that the two novices examined above developed outlines as extensive as those who had prompts, the process of outlining seemed to be an exercise rather than preliminary planning that guided their writing. The embedded prompts provided the novices that received them with additional support that was more directly connected to the generation of ideas in the text.

\section{Advanced Writers}

Advanced writers using the outliner also planned more than those with a word processor alone, but this did not result in better compositions. Why were these stu- 
dents who had taken advanced courses in writing not able to take advantage of these aids to improve their compositions?

Without prompts, advanced writers did not use their developing rhetorical skills while writing. None of the students in this group made any rhetorical plans during their sessions. Furthermore, their outlines were underdeveloped: Paula made an outline of 9 entries consisting of 19 words; Roger made an outline of 4 entries of 7 words; and Frank made a 19-word, 10-entry outline. None of these outlines contained much information, and they proved to be of little use during composition. Nor did these students use the advanced features of the outliner.

The uselessness of the outline is most apparent with Frank's experience. He made the following outline:

\author{
I. Introduction \\ A. Story about information \\ B. Backround $[s i c]$ information \\ II. Theme \\ III. Position \\ A. For \\ IV. Introduction of opposing view \\ A. Opponents \\ V. Refute position \\ VI. Conclusion
}

He refers to the outline only twice once he starts to write. He comes to the final paragraph:

[Writing] The conclusion to this is that the aforementioned paragraph . . . let's say paragraphs ... I can't get a word ... explains ... explains ... explains ... My mind is a blank. I cannot think of anything. Possibly because there is no air getting to my brain. My brain is cloudy. I can't even think of a word. Wish I had a thesaurus. [Rereading] The conclusion to this is that the aforementioned paragraphs ... uh, what ... the aforementioned paragraphs ... shows or tells ... I don't know ... shows or tells something . . . shows or tells ... not tells, but ... Ah, maybe this will do it. No, that's not even a word. Possibly, that ... The conclusion to this is that the aforementioned paragraph shows that ... that what? That what?

Advanced writers without prompts behaved more like novices. They did not respond to the assignment as a rhetorical task, and they did not use their developing rhetorical skills.

In fact, the writers using Acta and embedded prompts in the larger study wrote the worst essays of all the advanced writers. An analysis of the protocols and products of advanced writers indicates that this is due to a dissonance, or lack of fit, between the way these aids work together and the skills that advanced writers bring to the task.

Prompts increased the planning of advanced writers who received them. However, the prompts serve a different function for advanced writers. While the embedded topical prompts served an important role in helping novices generate and organize the content of their essays, the reader and goal prompts evoked the developing rhetorical skills these writers had acquired in their advanced writing courses, skills that were otherwise unused without the prompts.

For example, Al opened an Acta window and moved back and forth easily from the prompts to the outline, responding to all the questions, including the reader and goal prompts. He created a long outline of 24 entries with three levels of subordi- 
nation and 102 words. These were the resulting sections of the outline constructed in response to the Reader and Goal prompts:

V. Intended reader would be anyone interested in the topic of surrogate mothers
A. Attitudes.
1. For
2. Against
3. Mixed (conditional)
B. Info reader may want.
1. Pros.
2. Cons.
3. Judge's decisions.
4. Talk with surrogate mothers and surrogate families

VI. Values, facts, examples from real life, etc. supporting my position

A. M.B.W. [Mary Beth Whitehead] trauma

VII. Contradictions

Once Al began the essay, he moved back and forth between the outline, the prompts, and the essay five times. The prompts and the outline served to stimulate the development of ideas and as a checklist to evaluate his progress. For example:

\begin{abstract}
[Having written five paragraphs supporting the Judge's decision, he returns to his outline and reads:] um attitudes ... dealt with . . . info . . .that's almost dealt with, we'll deal with that in the conclusion. Contradictions ... okay, the rest of the story ... . IMoving to the prompts and reading reader and goal prompts:] What would people most want to know from your writing? ... Considering your answers to questions about the topic ... Your essay will be persuasive ... Convince the reader of your position . . . strategies . . . okay . . . Now I should look at the other side of the issue. [Al goes on to address and refute the opposing position.]
\end{abstract}

The prompts and the outliner worked well together for Al. However, the experience of a second advanced student using the outliner, Joe, illustrates the potential problems prompts have for advanced writers. As useful as the prompts were for some students, they seemed to be too intrusive for this student. Joe gave the impression of being very frustrated and angry. He swore frequently and did not like answering the prompts. As soon as he read the first prompt, he reacted belligerently: " What is this, they're teaching me how to write here?" He went on to say, "But this is not the way I form outlines . . . 'cause they're just like making you fill out facts here, and then, I'm gonna have to bring up another Acta card to make my real outline."

Ultimately, Joe went on to respond extensively, though begrudgingly, to all of the prompts, entering 449 words. In addition, he created a second outline of 14 entries and 141 words that focused primarily on the topic. It was this second, content-oriented outline to which Joe referred while writing. He did not reference the prompts or the responses on which he worked so hard but disliked so much, and none of the rhetorical plans contained in the prompt responses or outline were used during composition. For example, one of the responses to a goal prompt was: "The paper would be most effective through logical emotional argument." There was no representation of this or any other goal or audience responses in the second, content-based outline. Consequently, these rhetorical strategies were not used when the final text was written.

For the first of these subjects, the prompts served to evoke rhetorical thinking that would not have occurred otherwise, based on the experience of advanced writers without prompts. The second subject also responded fully to the prompts, 
including the reader and goal prompts. But because the prompts were dissonant with the way he normally wrote, this subject created a second, content-oriented outline that inhibited, rather than facilitated, access to rhetorical plans contained in the prompt responses. While the prompts increased the amount of planning for this student, the outliner served to disconnect or interfere with the movement between planning and composing.

\section{Difficulties Using the Software}

In general, students did not have difficulty using the software, and this did not appear to be a factor in the study. Only $4.1 \%$ of all protocols were coded as expressing difficulty with using the software. Actually, most of this can be attributed to the use of the prompts. While $7.0 \%$ of the protocols of students who received prompts expressed difficulty, only $1.2 \%$ of the protocols were expressions of difficulty by students who reccived the outliner without prompts.

\section{SUMMARY AND CONCLUSIONS}

The planning of writers in this study increased with use of the outliner and the embedded prompts. But it was only for novices who used the two aids together that this increased planning resulted in better compositions. This combination of the outliner and the prompts helped novices organize information and use it effectively in their essays. Without the prompts, the outliner did not provide enough support to enable the increased planning to result in improved compositions for novice writers. Furthermore, not all of the prompts benefited the novices; the reader and goal prompts were not useful to these writers. Tools that leave novice writers to their own resources or those that attempt to evoke skills that these writers do not have provide insufficient support to improve writing.

This emphasizes the need for pedagogical assistance (computer-based or otherwise) to fall within what Vygotsky (1978) calls the zone of proximal development - the difference between what learners can do on their own and what they can do with the guidance of experts or more experienced peers (or computer software). While reader and goal prompts appeared to be outside the zone of proximal development of novice writers, the topic prompts helped them think through their positions on the topic and plan their essays. The outliner helped them to structure and organize these ideas.

Advanced writers, on the other hand, benefited from the reader and the goal prompts as well. Thus, the use of prompts embedded in the outliner increased the planning of the advanced writers, particularly their rhetorical planning. Apparently, the reader and goal prompts evoked skills that these students had acquired in their advanced writing classes, skills not yet used automatically. However, for one student, these prompts conflicted with skills that had been internalized. It illustrates that certain pedagogical methods may conflict with learners' preferred strategies and reduce performance (Snow \& Lohman, 1984). Apparently, instructional support can be dysfunctional beyond either end of the zone of proximal development.

As for the use of the outliner, there seemed to be little systematic difference between the outlines of novices and those of the advanced students or their uses of the outliner. Of the five novices, three created well-developed and lengthy outlines, while the other two created brief outlines. The same division occurred among the 
five advanced students. There seemed to be no clear preference among the novices for topic outlines or sentence outlines. However, more of the advanced writers wrote topic outlines than sentence outlines.

All of the students used the most basic features of Acta, making "sisters" (parallel ideas), "aunts" (superordinate ideas), and "daughters" (subordinate ideas). A few changed the positions of entries by block moves. There were no indications in the protocols that the special collapse and expand features were used at all. While the most complex outline had 28 entries with four levels of subordination, the mean number of entries was 11 , with a mean of two levels of subordination. Since most students did not develop a great deal of subordinated detail in their outlines, there may have been little need to simplify the blocks of content. Use of the more advanced features of the outliner may come only with more complex tasks and extended use of the software.

\section{LIMITATIONS OF THE STUDY}

There are several considerations that limit the generalizability of the results. The assignment required the students to write an essay on a given topic. While the topic is typical of argumentative assignments, more often than not students in regular writing classes are able to select their own topic. In such a situation, it may be more likely that students select a topic about which they know more or in which they have greater personal interest. Furthermore, the study was conducted outside the context of a regular course. Thus, the task lacked other motivational aspects, such as concern about grade performance, the development of writing skills, and an interest in writing for others in the class, including the teacher. All of these constraints are likely to limit the amount of effort put into the writing process and the quality of the final product.

Also, the study required students to compose the essay in one sitting without access to reference materials. This quite likely limited the length and complexity of the essay and thus, perhaps, the need to use advanced features of the outliner. Extended sessions with access to resources may result in more information generated during initial planning. This may create a need to expand and contract the outline in order to consider the high-level structural issues for the argument. The advanced features of the outliner may make greater contributions to effective planning for more complex tasks.

Finally, the students in this study also had limited exposure to the tool. While the students appeared to have little difficulty in using the basic features of the outliner, use of the more advanced features may require more than the one practice session used in this study. Subsequent research should examine the developing use of outliners over multiple exposures and a range of tasks.

\section{IMPLICATIONS FOR CLASSROOM USE}

This study clearly suggests that student planning benefits from the use of an outliner and embedded prompts. It also describes the difficulties some students have in effectively connecting these plans to their compositions. Such issues can be direct- 
ly addressed in the writing classroom, rather than leaving students to their own resources while using these tools to write.

For example, a teacher can introduce the use of an outliner within the context of structural planning, particularly the hierarchical ordering of ideas. The focus here is on the planning process, rather than the form of the outline. Part of the planning process is the consideration of whether an idea is primary or supportive of another idea, so therefore the students must consider the relationship of new ideas they generate and those previously written. The use of the outliner would be a natural expression of these hierarchical considerations. Students who have already had some experience in considering structural issues might use an outliner to gain a clearer view of their writing as a coherent structure and thus encounter fewer difficulties in connecting their plans to their compositions.

Other practices should be observed in the classroom that can increase the impact of these tools. Before introducing an outliner, the instructor should be sure that the students are thoroughly familiar with the word processor. Interference in the writing process may result from difficulties in learning to use both the word processor and the outline at the same time. Subsequently, students may also need extended practice with the outliner before they take advantage of its more powerful features.

The use of prompts embedded in the software, particularly topical prompts, can provide additional guidance that novice writers need. Advanced writers can also benefit from topical prompts, as well as those that consider audience and goal. However, it is important to remember that some of these students may find prompts are condescending or that they obstruct their own preferred approaches.

The mere introduction of a writing tool into the classroom will not be a panacea; they will work for some students and not others. The more that is known about the benefits as well as the limitations of writing tools such as outliners, the more effectively teachers will be able to integrate them into the writing curriculum in a way that meets the particular needs of their students.

Acknowledgment - The second author would like to acknowledge support from the Dana Foundation Fellowship for Computer-Assisted Instruction in the Humanities, at Carnegie Mellon University.

\section{REFERENCES}

Bereiter, C., \& Scardamalia, M. (1987). The psychology of written composition. Hillsdale, NJ: Erlbaum.

Brown, A., \& Palinscar, A. (1989). Guided, cooperative leaming and individual knowledge acquisition. In L. Resnick (Ed.), Knowing, learning, and instruction (pp. 393-454). Hillsdale, NJ: Erlbaum.

Emig, J. (1971). The composing processes of twelfth graders. NCTE Research Report No. 13. Urbana: NCTE.

Flower, L., \& Hayes, J. (1981). A cognitive process theory of writing. College Composition and Communication, 32, 365-387.

Fowler, H. (1980). The Little, Brown handbook. Boston: Little, Brown \& Co.

Gee, T. (1984). Drafting and revising processes in grade twelve students' examination writing. (ERIC Document Reproduction Service No. ED 269 770)

Glaser, B. (1978). Theoretical sensitivity. Mill Valley, CA: Sociology Press.

Glaser, B., \& Strauss, A. (1967). The discovery of grounded theory. New York: Aldine.

Haas, C. (1989). How the writing medium shapes the writing process: Effects of word processing on planning. Research in the Teaching of English, 23, 181-207.

Hayes, J., \& Flower, L. (1980). Identifying the organization of writing processes. In L. Gregg \& E. W. Steinberg (Eds.), Cognitive processes in writing (pp. 3-30). Hillsdale, NJ: Erlbaum. 
Kellogg, R. (1987). Writing performance: Effects of cognitive strategies. Written Communication, 4, 269-298.

Kozma, R. (1991a). Computer-based writing tools and the cognitive needs of novice writers. Computers and Composition, 8(2), 31-45.

Kozma, R. (1991b). The impact of computer-based tools and embedded prompts on writing processes and products of novice and advanced college writers. Cognition and Instruction, 8, 1-27.

Kozma, R. (1991c). Leaming with media. Review of Educational Research, 61, 179-211.

Salomon, G., Perkins, D., \& Globerson, T. (1991). Partners in cognition: Extending human intelligence with intelligent technologies. Educational Researcher, 20(3), 2-9.

Snow, R., \& Lohman, D. (1984). Toward a theory of cognitive aptitude for learning from instruction. Journal of Educational Psychology, 76, 347-376.

Vygotsky, L. (1978). Mind in society. Cambridge, MA: Harvard University Press.

Wresch, W., Pattow, D., \& Gifford, J. (1988). Writing for the 21 st century: Computers and research writing. New York: McGraw-Hill.

\section{APPENDIX A}

\section{EMBEDDED PROMPTS}

\section{Your Directions}

First, generate ideas by answering some questions about the topic you are given, then plan how those ideas might fit together into a purposeful writing, appropriate for a specific reader. After you answer these questions, write an essay of as much as you think needed on the topic you have been assigned.

\section{Your Topic}

This is your topic: (Assignment)

What do you know about (Assignment)?

List facts, events, people, places, etc. that are relevant to the topic:

Make a list of facts or reasons why this decision might be a good idea.

Make a list of facts or reasons why this decision might not be a good idea.

Your position.

What is your position on (Assignment)?

\section{Your Reader}

Considering your answers to the questions about the topic, who do you think would be most interested in your comments?

What attitudes could this reader be expected to have toward the topic?

What attitudes could this reader be expected to have toward your position?

\section{Your Goal}

Since you will be expressing your opinion about the topic, your essay will be persuasive. You will want to convince your reader to agree with your position. How do you think you might motivate the reader to agree with you?

One strategy you can use in your paper is to state your position and then give some values, facts, examples from real life, etc. that support it. What are these? 
Another strategy is to give some values, facts, examples from real life, etc. that contradict the position of your reader, if it is other than yours. What are some of these?

List the values, facts, examples that would most convince your reader.

\section{Your Paper}

Considering the responses to the various questions, write your essay below. You may go back to read or copy anything you have written. You may write as much as you think necessary.

\section{APPENDIX B}

\section{ACTA CHECKLIST}

- Opening Acta

- Understanding the family structure of Acta

- Entering a subtopic by selecting New Daughter from the Acta menu

- Double-clicking the triangle to expand a topic (show all subtopics) or to collapse a topic (hide all subtopics)

- Moving a topic by changing its position in the family

- Moving a topic and its subtopic with the mouse

- Deleting a topic

- Learning window movements

- Cutting and pasting in Acta the same way as in MacWrite using the Edit menu

- Moving quickly between Acta and MacWrite 